\title{
CALCULATIONS OF STM LINESCANS - GENERAL FORMALISM
}

\author{
Ş. Ellialtıŏlu \\ Physics Department \\ Middle East Technical University \\ Ankara 06531, Turkey \\ S. Ciraci \\ Department of Physics \\ Bilkent University \\ Maltepe, Ankara 06572, Turkey \\ Inder P. Batra \\ IBM Research \\ Almaden Research Center \\ 650 Harry Road \\ San Jose, California 95120-6099
}

(Received 18.02 .88 by P.H. Dederichs)

\begin{abstract}
We have developed a formalism for calculating the line scans of the scanning-tunneling microscopy from the realistic substrate and tip wave functions. The tip wave functions are calculated self-consistently by using a spherical jellium corresponding to a particular metal with various radii. This formalism provides a framework to analyze the experimental line scans, and to deduce information about the clean and adatom covered surfaces, and the radius and height of the tip, as well. We have found that the contribution of a tip wave function in tunneling current is strongly dependent on its symmetry.
\end{abstract}

The pioneering work of Binnig et.al.[1] has introduced the Scanning Tunneling Microscopy (STM) technique, which directly ensures the real-space determination of the surface structure of a sample. Thus, the periodicity of the sample surface is not a requirement for the investigation. So far, it has been demonstrated that STM technique is able to detect the isolated steps, dislocations and even single vacancies or impurities at the surface. In one of the modes of operation in STM, the tip height is adjusted to keep the tunneling current constant at a fixed bias. The interpretation was that the tip retracts at the high charge density points, indicating the positions of the atoms at the surface.

A formalism has been developed by Tersoff and Hamann[2] using frozen wavefunctions of both electrodes in the Bardeen's expression[3] for the tunneling current. They have assumed an idealized tip with con- stant density of states, and the decay of the charge density to be purely exponential with exponents determined from the sample workfunction. The tunneling current was found to be proportional to the local density of states of the substrate at the Fermi level evaluated at the tip position, $\rho_{s}\left(\vec{R}, E_{F}\right)$.

According to Tersoff and Hamann's theory[2] the constant current contours observed with STM are actually contours of constant $\rho_{s}\left(\vec{R}, E_{F}\right)$ of the sample at $\vec{R}=(\vec{\tau}, \vec{\xi})$ where $\vec{\tau}$ is the lateral position vector in the substrate surface, and $\vec{\xi}=\left(r_{0}+h\right) \hat{z}, r_{0}$ being the radius of the tip (assumed to be spherical), and $h$ is the tipto-surface distance. In the earlier studies, one has generated usually the contour plots from the line scans obtained from the constant current-constant voltage mode in STM, and has compared these with the calculated charge density plots. The surface charge densities have 
been calculated by using various methods within the repeating slab geometry, and the Tersoff and Hamann's theory has been applied with reasonable success. For example, in the recent experimental observations[4] on graphite, STM was able to resolve the two inequivalent atoms. This was a very significant observation since the only distinction between these two sites is caused by a weak interlayer coupling of the graphite planes. A correct identification of these different atomic sites in graphite has been achived recently by using the charge density calculations by Batra et al.[5], and also by Selloni et al.[6].

It should be noted, however, that many fine structures in the line scans- which are possibly of crucial importance- are lost in generating the contour plots. In this respect, the analysis of a line scan itself would reveal additional information about the surface, which is not so clear in the contour plots. It is, therefore, desirable to calculate a quantity starting from the wavefunctions of the sample and the tip, which can bc compared with the STM line scans. In this study, we present a formalism for calculating the STM line scans. This is based on the direct use of the substrate wave functions, which can be obtained from the SCF-pseudopotential calculations[7]. The extension to other forms of the wave functions, for example, the LCAO wave functions is straight forward. Since the realistic tip wave functions can be obtained from the SCF- calculations of the spherical jellium metal, the effects of the symmetry of the tip wave functions on the tunneling current are also included.

Slab wave functions are calculated with a reasonable accuracy within a region relevant to the tip-tosurface distance one usually gets in STM. Certainly these wave functions are more realistic than those exponentially decaying empirical forms with exponents obtained from the workfunction data. In general, the calculated slab wave functions have the form

$$
\phi_{n}\left(\vec{k}_{\|}, \overrightarrow{r^{\prime}}\right)=\sum_{\vec{G}} a_{n}\left(\vec{k}_{\|}+\vec{G}\right) e^{i\left(\vec{k}_{\|}+\vec{G}\right) \cdot \overrightarrow{r^{\prime}}}
$$

where $a_{n}$ are the expansion coefficients for each band $n$, and $\vec{k}_{\|\|}$is the wave vector parallel to the surface. Vectors $\vec{G}$ are the 3-dimensional reciprocal lattice vectors of the slab unit cell, which contain the vacuum region between two slabs. $\vec{r}$ is the 3-D position vector of the substrate.

Since the structure of a tip is usually not known in STM, we simulate it with a spherical-jellium metal. Within this model, the wave functions of the tip are computed self-consistently as a function of the tip radius. Jellium model has been applied to various systems so far, including semi-infinite slabs representing a metal surface[8], slabs of finitc thickncss[9], and small spheres representing metal clusters $[10,11]$. It was observed for the jellium ball that the electronic energy levels obey the shell structure and one gets new solutions for the energy eigenstates accordingly, as the radius of the sphere increases. The corresponding charge density when solved self-consistently for a given radius exhibit the usual Friedel oscillations and a tail representing the electrons spilling out of the sphere. Figure 1 schematically shows the self-consistent solutions for two jellium spheres of different radii. The self-consistent potential $V_{\text {eff }}$ is the sum of the Hartree and exchange-correlation terms[12]. Jellium results show that the tails of the charge density, $n_{t}(\vec{r})$ for spheres of different radii do not change very much, whereas $n_{t}(\vec{r})$ at $r=0$ changes drastically as one increases the radius, $r_{0}$, to include especially a new s- or p-type level each time. The wave functions calculated numerically are then cast in an analytical form as linear combinations of Gaussian (GTO) or Slater (STO) type orbitals. This way not only the s-symmetry, but also different other symmetries for the tip functions can be investigated separately. The GTO's we have considered are expressed as :

$$
\begin{gathered}
\psi_{0}(r)=\sum_{i} c_{i 0} e^{-\alpha_{i 0} r^{2}} \\
\psi_{1}(r)=\sum_{i} c_{i 1} e^{-\alpha_{i 1} r^{2}}\left\{\begin{array}{l}
r \cos \theta \\
r \sin \theta \cos \phi
\end{array}\right\}
\end{gathered}
$$

for the s-, $p_{z}-$ and $p_{x}$-type wave functions, respectively. The angular part for $\mathrm{p}_{y}$-type wave function is $\sin \theta \sin \phi$. Similar expressions for the corresponding STO's are :

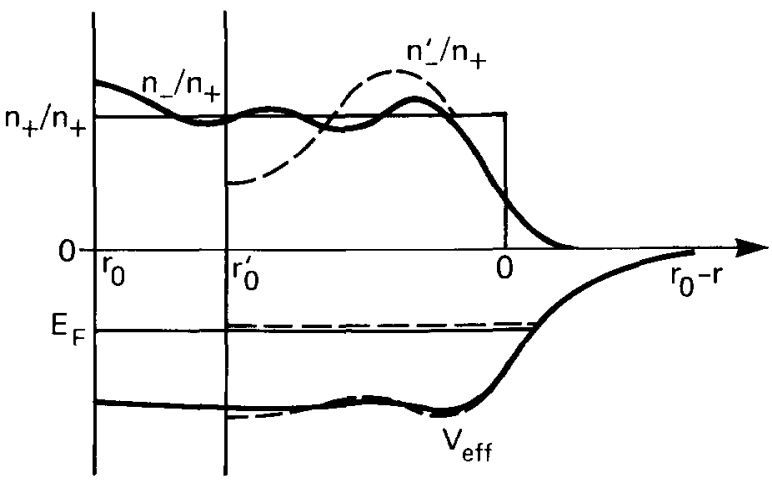

Figure 1. Schematic illustration of jellium results for effective potentials and the normalized densities of electron states of two metal spheres with different radii $\left(r_{0}^{\prime}\right.$ and $\left.r_{0}\right)$. The density of electron states at the interior regions, especially at the center, are quite different, but the tails at the exterior region are very similar. 


$$
\begin{gathered}
\psi_{0}(r)=\left(\frac{\alpha_{0}}{2 \pi}\right)^{1 / 2} \frac{e^{-\alpha_{0} r}}{r} \\
\psi_{1}(r)=\left(\frac{3 \alpha_{1}^{3}}{\pi}\right)^{1 / 2} e^{-\alpha_{1} r}\left\{\begin{array}{l}
\cos \theta \\
\sin \theta \cos \phi
\end{array}\right\}
\end{gathered}
$$

The tunneling current is given by the first order perturbation theory as[2,3]

$I=\frac{2 \pi}{\hbar} \sum_{l} \sum_{n} f\left(E_{l}\right)\left[1-f\left(E_{n}+e V\right)\right]\left|M_{l n}\right|^{2} \delta\left(E_{l}-E_{n}\right)$

where $V$ is the applied bias voltage. $M_{l n}$ are the tunneling matrix elements which can be written in Bardeen's formalism as

$$
M_{l n}=\frac{\hbar^{2}}{2 m} \int_{0} d \vec{S} \cdot\left(\phi_{n}^{*} \vec{\nabla} \psi_{l}-\psi_{l} \vec{\nabla} \phi_{n}^{*}\right)
$$

where $S$ is a surface in the vacuum region sufficiently far from the tip and slab. This formalism assumes frozen wave functions $\phi_{n}$ and $\psi_{l}$ representing the two electrodes whose separations are large enough to be treated independently. However, as shown by Tekman and Ciraci[13], the frozen orbital approximation is not justified at small tip-to-surface distances. For the integration surface $S$, we have chosen a spherical shell concentric with the jellium ball shown in Fig.2. With this simple choice of surface geometry and the variables described in Fig. 2, the matrix elements $M_{l n}$ can be put into a compact analytical form in terms of a function $B_{l}(Q)$, which is independent of the tip position :

$$
M_{l n}=\frac{\hbar^{2}}{2 m} \sum_{\vec{G}} a_{n}(\vec{Q}) e^{i \not{Q} \cdot \vec{R}} B_{l}(Q)
$$

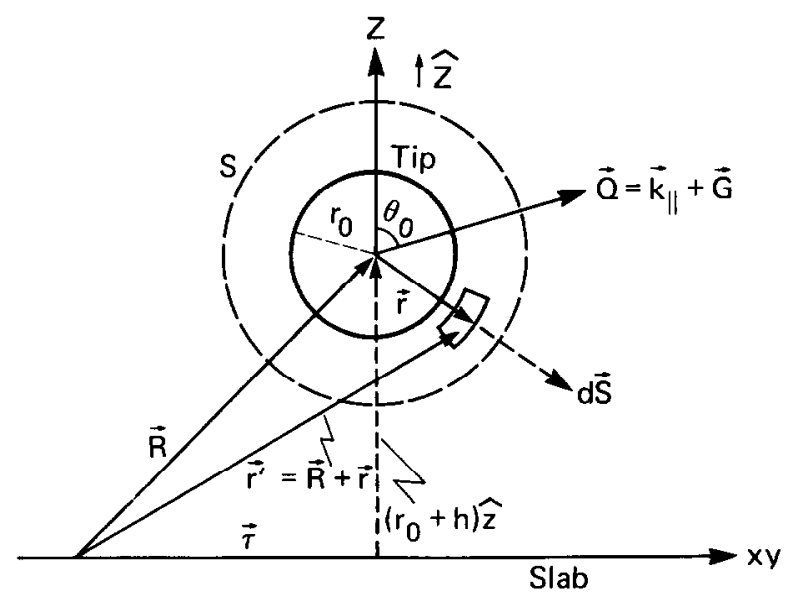

Figure 2. The geometry of the electrodes based on the analytical calculations. The tip is represented by a sphere of radius $r_{0}$, and concentric with that is the surface of integration of spherical shape of radius $r_{0}<r<r_{0}+h$.
Here $\vec{Q}=\vec{k}_{\|}+\vec{G}$ and the functions $B_{l}(Q)$ are to be calculated once for all, and are given for different types of analytical tip wave functions as :

$$
\begin{gathered}
B_{0}(Q)=4 \pi \sum_{i} c_{i 0} e^{-\alpha_{i 0} r^{2}}\left[2 \alpha_{i 0} r^{2} j_{0}(Q r)-Q r j_{1}(Q r)\right] \\
B_{1}(Q)=4 \pi \sum_{i} c_{i 1} e^{-\alpha_{i 1} r^{2}}\left[\left(2 \alpha_{i 1} r^{3}-r-2\right) j_{1}(Q r)+Q r j_{0}(Q r)\right] \\
\times\left\{\begin{array}{c}
r \cos \theta_{0} \\
r \sin \theta_{0}
\end{array}\right\}
\end{gathered}
$$

for the s-type and $\mathrm{p}_{z}, \mathrm{p}_{x, y}$-type GTO's, respectively. Similarly for the corresponding STO's one obtains :

$$
\begin{gathered}
B_{0}(Q)=(8 \pi)^{1 / 3} \alpha_{0} e^{-\alpha_{0} r}\left[\left(\alpha_{0} r+1\right) j_{0}(Q r)-Q r j_{1}(Q r)\right] \\
B_{1}(Q)=(48 \pi)^{1 / 3} \alpha_{1} e^{-\alpha_{1} r}\left[\left(\alpha_{1} r-2\right) j_{1}(Q r)+Q r j_{0}(Q r)\right] \\
\times\left\{\begin{array}{l}
r \cos \theta_{0} \\
r \sin \theta_{0}
\end{array}\right\}
\end{gathered}
$$

where $j_{l}(Q r)$ are the spherical Bessel functions. If one assumes a concentric hemispherical shell for the surface $\mathrm{S}$ in Fig.2, then the above results will be in terms of the spherical Hankel functions $h_{l}^{(1)}(Q r)$ instead. In spite of the fact that the radius of the surface of integration $S$, appears explicitly in the above equations, $B_{l}(Q)$ 's are $r$-independent. To check this analytically as well, we have taken the limit of Tersoff and Hamann's[2] in our formalism and obtained their result identically.

Having obtained the above expressions the calculations of line scans are straihtforward. For a given value of the tip-to-slab distance $h$, where $\vec{R}=\left(h+r_{0}\right) \hat{z}+\vec{\tau}$, one generates an $\mathrm{I}(\vec{\tau})$ ver sus $\vec{\tau}$ curve $C$. For several fixed values of $h_{i}$ one repeats the same scan, and obtains a family of curves $C_{i}$ of $I(\vec{\tau})$ versus $\vec{\tau}$. An illustration of this is shown in Fig.3. For a constant value $I_{0}$ of the

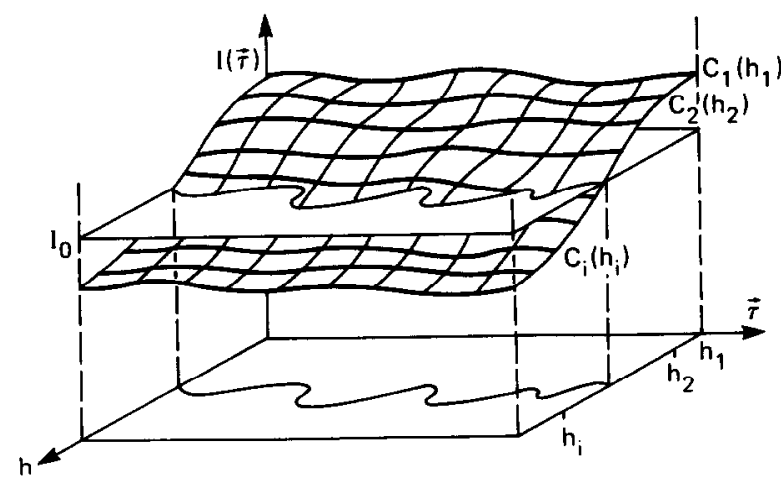

Figure 3. Family of constant-height curves $\mathrm{C}_{i}\left(h_{i}\right)$ displaying the current $I(\vec{\tau})$, versus lateral position $\vec{\tau}$ schematically. The projection of the interception of the constant $I_{0}$ plane with the surface composed of $C_{i}$ 's is the corrugation $\mathrm{h}(\vec{\tau})$ of the slab surface at constant $\mathrm{V}$ and constant $\mathrm{I}_{0}$. 
current shown by the horizontal plane, one obtains the corrugation $h(\vec{\tau})$ graphically.

It should be noted that the jellium ball used in the present analysis has a discrete set of eigenstates. Whereas the continium of electronic levels of an actual tip leads to a state density $D(E)$, which ought to amplify the tunneling current. The tip wave functions, $\psi$, obtained from the jellium ball are good representations for those participating in the actual tunneling event, except that they are normalized with respect to the volume of the idealized spherical tip. As a result the observed tunneling current has to differ from that calculated here by a constant multiplicative factor. However, $(\mathrm{dI} / \mathrm{dh})_{V}$ within the conventional tip-to-surface distance, $h$, is expected to be unaltered both in theory and in experiment. In view of the above arguments the calculated line scans can be compared safely with the experimental ones. From that comparison, the tipto-surface distance can be estimated. Since the family of curves $C_{i}$ is easily generated for various tip radii, the effect of the tip radius can also be revealed. Furthermore, using single exponent STO to represent the valence orbitals of the tip atoms and simulating the atomic structure at the tip by small radius jellium balls, the effect of the atomic structure of the tip (i.e. tips having one, two or more outermost atoms) on the STM images can be investigated within the formalism we developed. We found also the following significant effects of the tip wave function symmetry on the tunneling current: (i) Tips with $\mathrm{p}_{x^{-}}$and $\mathrm{p}_{y^{-}}$-type wave functions are sensitive to the xy-corrugation only. They are less sensitive to the changes in the vertical direction. (ii) Tips with a $p_{x}$ -type wave function are less sensitive to $x y$-corrugation. Therefore, since the contributions to the tunneling current from different types of wavefunctions are not equal, in contrast to earlier proposal[2], the current is not necessarily just proportional to the total state density of te tip at the Fermi energy. Furthermore, each of these unequal contributions is not directly proportional to the corresponding local density of states, either. Apparently, the symmetry of the tip wave function plays an important role in the interpretations of the experimental data.

It should also be noted that using the precalculated functions $B_{l}(Q)$, we can calculate not only the constant current-constant voltage mode, but also other quantities like the conductance $(\mathrm{dI} / \mathrm{dV})_{h}$, and the effect of the vertical motion of the tip on the current, $(\mathrm{dI} / \mathrm{dh})_{7}$. Since the wavefunctions for the adatom covered (monoor multilayer coverage) metal and semiconductor surfaces can be accurately calculated by using the SCF-slab calculations, the STM line scans for such systems can be investigated within the formalism presented here. In the matrix element $M_{l n}$, the type of the adatom is characterized by the Fourier transforms of the wave function $a_{n}(\vec{Q})$. Hence, the matrix elements $\mathrm{M}_{l n}$ and the line scans have to contain the information, wherefrom the signature of the adatom can be deduced.

\section{ACKNOWLEDGEMENT}

We would like to thank Professor M. Durgut for helpful discussions.

\section{References}

[1] G. Binnig, H. Rohrer, Ch. Gerber and E. Weibel, Appl. Phys. Lett. 40, 178 (1982); Phys. Rev. Lett. 49, 57 (1982); Phys. Rev. Lett. 50, 120 (1983); Surf. Sci. 131, L379 (1983); G. Binnig and H. Rohrer, Surf. Sci. 126, 236 (1983).

[2] J. Tersoff and D.R. Hamann, Phys. Rev. Lett. 50, 1998 (1983); J. Tersoff and D.R. Hamann, Phys. Rev. B31, 805 (1985).

[3] J. Bardeen, Phys. Rev. Lett. 6, 57 (1061).

[4] G. Binnig, H. Fuchs, Ch. Rohrer, E. Stoll and E. Tosatti, Europhys. Lett. 1, 31 (1985).
[5] I.P. Batra, N. Garcia, H. Rohrer, H. Salemink, E. Stoll and S. Ciraci, Surf. Sci. 181, 126 (1987); H. Salemink, I.P. Batra, H. Rohrer, E. Stoll and E. Weibel, Surf. Sci. 181, 139 (1987); I.P. Batra, S. Ciraci, N. Garcia, H. Rohrer, H. Salemink and E. Stoll, in 18th. Semiconductor Conf. Proc., Stockholm, 1986.

[6] C.A. Selloni, P. Carnevalli, G.D. Chen and E. Tosatti, Phys. Rev. B31, 2602 (1985).

[7] M. Schluter, J.R. Chelikowsky, S.G. Louie and M.L. Cohen, Phys. Rev. B12, 4200 (1975). 
[8] N.D. Lang and W. Kohn, Phys. Rev. B1,4555 (1970); Phys. Rev. B3, 1215 (1971).

[9] F.K. Schulte, Surf. Sci. 55, 427 (1976).

[10] W. Eckard, Phys. Rev. B29, 1558 (1984).

[11] D.E. Beck, Solid State Commun. 49, 381 (1984).
[12] W. Kohn and L.J. Sham, Phys. Rev. 140, A1133 (1965).

[13] E. Tekman and S. Ciraci, Physica Scripta, $\mathbf{x x}, \mathbf{x x x x}$ (1988). 